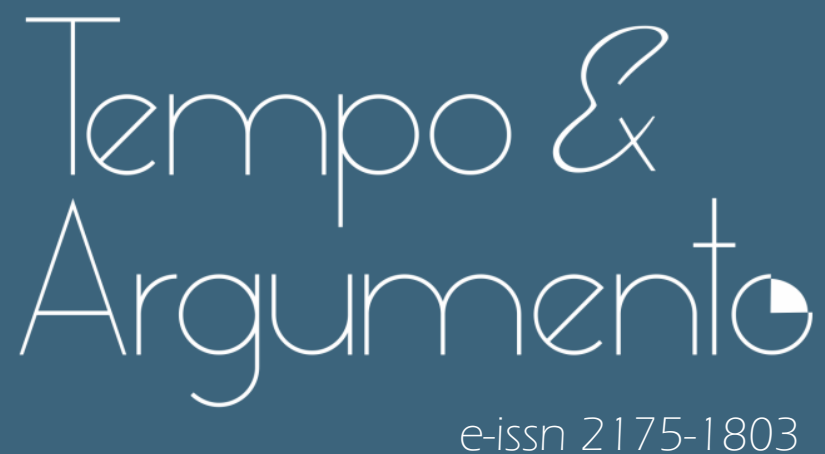

Triple presencia femenina en torno a los trabajos: mujeres de sectores populares, participación política y sostenibilidad de la vida

E Juliana Díaz Lozano

Doctora en Ciencias Sociales por la Universidad Nacional de La Plata (UNLP).

Becaria Posdoctoral del Consejo Nacional de Investigaciones

Científicas y Técnicas (CONICET).

La Plata - ARGENTINA

http://mww.memoria.fahce.unlp.edu.ar/perfiles/1933DiazLozanoJ.html

diazlozano.juliana@gmail.com

(iD) orcid.org/0000-0000-0000-0000

Para citar este articulo:

DÍAZ LOZANO, Juliana. Triple presencia femenina en torno a los trabajos:

mujeres de sectores populares, participación política y sostenibilidad de la vida.

Tempo e Argumento, Florianópolis, v. 12, n. 29, e2001, 2020.

doi http://dx.doi.org/10.5965/217518031229202001

Recebido: 09/09/2019

Aprovado: 14/11/2019 


\title{
Triple presencia femenina en torno a los trabajos: mujeres de sectores populares, participación política y sostenibilidad de la vida
}

\begin{abstract}
Resumen
Este artículo analiza las experiencias de mujeres que integran organizaciones barriales populares en relación con el trabajo. Para ello, se parte de reconstruir las condiciones de vida de las mujeres trabajadoras, de sectores populares y racializadas en las periferias argentinas, tomando en consideración, entre otros elementos, la propuesta estatal de políticas sociales y vinculadas al trabajo para este sector. Para abordar las experiencias laborales de las mujeres, se recuperan los aportes de la Economía Feminista sobre la ampliación de la categoría trabajo, poniendo la sostenibilidad de la vida en el centro. Desde esta óptica, se considera la totalidad del trabajo realizado por las mujeres como un continuo, que entrelaza diferentes actividades remuneradas, de cuidados o reproductivo y comunitario, a fin de sostener sus vidas y las de sus familias. A partir de analizar la totalidad de la experiencia cotidiana femenina y los usos del tiempo que realizan en los distintos ámbitos de desempeño, discutimos la pertinencia de la idea de jornadas laborales; postulamos, en cambio, la mayor utilidad del concepto de doble presencia femenina; y afirmamos la existencia de una triple presencia para las mujeres militantes.
\end{abstract}

Palabras clave: Mujeres. Trabajo. Cuidado. Participación Política. Triple Presencia.

\section{Triple female presence around the works: women from popular sectors, political participation and life sustainability}

\begin{abstract}
This article analyzes the experiences of women who are members of popular neighborhood organizations in relation to work. To do this, we start by reconstructing the living conditions of working women, from popular sectors and racialized in the Argentine outskirts, taking into account, among other elements, the State proposal of social and labor-related policies for this sector. To address women's labor experiences, the contributions of Feminist Economy to broaden work as a category are resumed, putting life sustainability at the center. From this perspective, all the work carried out by women is regarded as a care or reproductive and community continuum, which intertwines various paid jobs, in order to provide for their individual and family living expenses. By analyzing the daily female experience as a whole and their uses of time in their various working areas, we discuss the relevance of the idea of working hours; we assume, instead, the greater usefulness of the concept of double female presence; and we claim the existence of a triple presence for militant women.
\end{abstract}

Keywords: Women. Work. Care. Political Participation. Triple Presence. 


\section{Introducción}

Este artículo analiza las experiencias de mujeres que integran organizaciones barriales populares en relación con el trabajo. Para ello, se parte de considerar las condiciones de vida de las mujeres trabajadoras, de sectores populares y racializadas en las periferias argentinas, que constituyen la base social mayoritaria de las citadas organizaciones. En esta caracterización también se incluye la propuesta estatal de políticas sociales para este sector. Para abordar las experiencias laborales de las mujeres, se recuperan los aportes de la Economía Feminista sobre la ampliación de la categoría trabajo, poniendo la sostenibilidad de la vida en el centro (PÉREZ OROZCO, 2014; RODRÍGUEZ ENRÍQUEZ, 2015; CARRASCO, 2017). Desde este enfoque, se considera la totalidad del trabajo realizado por las mujeres como un continuo, que entrelaza diferentes actividades remuneradas, de cuidados o reproductivo y comunitario, a fin de sostener sus vidas y las de sus familias. Esta enorme carga de labor femenina fluctúa en función del contexto económico, social y político, y también de las prácticas individuales y colectivas desplegadas por las mujeres. Esta concepción del trabajo rompe su correspondencia con empleo, y la amplía a todo el conjunto de trabajos que las mujeres realizan, producto de una división sexual del trabajo patriarcal, incluyendo en un lugar central los trabajos que no son valorados socialmente como tales. A partir de analizar la totalidad de la experiencia cotidiana femenina y los usos del tiempo que realizan en los distintos ámbitos de desempeño, discutimos la pertinencia de la idea de jornadas laborales para postular, en cambio, la mayor utilidad del concepto de doble presencia femenina y afirmar la existencia de una triple presencia para las mujeres que además participan políticamente.

Este artículo es una derivación de la tesis de investigación Mujer Bonita es la que sale a luchar. Experiencias de vida de mujeres participantes del Frente Popular Darío Santillán Corriente Nacional de Berisso (no publicada), cuyo objetivo fue analizar cómo la participación política en organizaciones para la supervivencia atraviesa las vidas cotidianas y las biografías de las mujeres de sectores populares. El Frente Popular Darío Santilán Corriente Nacional (en adelante "el Frente") es una organización político-social de vocación 
anticapitalista y antipatriarcal surgida en 2004 como resultado de un proceso histórico que se renueva en las luchas contra las políticas neoliberales que se dieron con virulencia extrema en Argentina durante la década de 1990, momento donde se iniciaron los primeros movimientos de trabajadores desocupados (MTD). Estos colectivos nuclearon a una gran masa de trabajadores expulsados del mercado formal, pero también a mujeres y jóvenes que nunca fueron asalariados, pero ante la crisis y la pauperización, se vieron forzados a organizarse para buscar un sostén económico (CROSS; PARTENIO, 2004). Forma parte de las organizaciones llamadas piqueteras, que se destacan por su método, el piquete, o corte de ruta, como forma de protesta para obtener sus demandas del Estado y las empresas. Como parte de su labor, se cuenta la apertura de comedores populares, la creación de huertas y espacios educativos en los barrios, y la lucha por obtener la gestión colectiva de políticas sociales, como los programas de cooperativas de trabajo dependientes del Ministerio de Desarrollo Social.

El foco de este estudio en las experiencias de las mujeres del Frente - en tanto organización popular barrial - tiene que ver con que son ellas quienes constituyen las principales participantes (en número) y las dinamizadoras de las tareas diarias colectivas (p. ej., ANDÚJAR, 2014; CAUSA, 2007; CROSS; PARTENIO, 2004). El barrio como espacio de producción política de los sectores populares encuentra en las mujeres los artífices principales de los entramados comunitarios que lo dinamizan. La persistencia y prevalencia de las mujeres en las organizaciones populares barriales se relaciona con el hecho de que son las garantes de la mayor parte del trabajo de cuidados (PÉREZ OROZCO, 2014), por lo que su participación en esta u otras instancias colectivas supone - más allá de los cambios económicos - la posibilidad de reproducir su vida y la de su familia.

Por consiguiente, nos centramos en las experiencias de mujeres, en su gran mayoría madres, que se organizan para realizar trabajo comunitario y sostener su vida en común con otras personas. En relación con el recorte temporal, la gran parte del trabajo de campo fue realizada entre los años 2012 y 2017, a partir de entrevistas en profundidad a 20 mujeres y la observación de su desempeño en 3 de las asambleas barriales del Frente en Berisso, realizadas semanalmente 
en los comedores de esta organización en el barrio Villa Argüello: "Juanito Laguna"; "Los Amigos"; y "Madres Unidas". Como parte de esta participación, se integran a las cooperativas de trabajo financiadas a partir de programas estatales, que proveen un ingreso monetario mínimo, obtenido a partir de movilizaciones y gestiones por parte del Frente. La inserción en la cooperativa y la elaboración de alimentos en el comedor para sus familias y vecinos les permite, como ellas definen, "parar la olla"1.Como contraparte, la participación en el Frente implica para las mujeres el cumplimiento de una serie de "criterios" u "obligaciones recíprocas y compartidas" (LINSALATA, 2015), entre los(las) que se cuentan, el trabajo en el barrio y la participación en las asambleas barriales semanales (espacio de decisión) y en las movilizaciones callejeras.

Sus biografías están, además, atravesadas por experiencias de migración desde países cercanos u otras provincias, y en la mitad de los casos son jefas de hogar. Tienen diferentes tiempos de participación en la citada organización desde 1 año hasta alrededor de 10 - y todas tienen en común vivir en una zona de asentamiento informal en el barrio Villa Argüello, de la localidad de Berisso.

Este artículo está estructurado en 5 apartados. En el primero de ellos se caracterizan las condiciones de vida de las mujeres de sectores populares en las periferias urbanas de Argentina durante los años de la investigación, pero integrando las transformaciones económicas y sociales desde la década de 1990. En el segundo apartado, se describe y analiza la diversidad de actividades laborales realizadas por las mujeres como un continuo de trabajo cotidiano. En el tercer, se caracteriza al trabajo de participación comunitaria femenina. Y en los últimos 2 apartados se discute la pertinencia de la idea de jornadas laborales para describir el trabajo femenino y se postula la existencia de dobles y triples presencias laborales de las mujeres militantes de sectores populares.

\section{Condiciones de vida de las mujeres de sectores populares}

A partir de la década de 1990, se consolidan en Argentina las reformas neoliberales que provenían de la época dictatorial, con un viraje de una economía 
industrial a otra financiera, agropecuaria, y de servicios. El desmantelamiento del modelo de sustitución de importaciones y la privatización de las empresas públicas fueron elementos claves de la aplicación de esas políticas (SVAMPA; PEREYRA, 2003; PACHECO, 2002). Por otra parte, se produjo una desregulación del mercado de trabajo impulsada por los organismos financieros internacionales y la fracción dominante local (BASUALDO, 2008). Fueron los trabajadores quienes absorbieron buena parte de la crisis industrial, marcada por la baja de salarios, la polarización, y la concentración sectorial alrededor de las grandes firmas oligopólicas. Con la privatización de las empresas estatales, muchos trabajadores perdieron sus empleos, otros sufrieron la precarización y algunos comenzaron el camino de la supervivencia, a partir de trabajos temporales que les permitieran comer diariamente.

En el caso de las mujeres, la profundización de las políticas neoliberales significó, entre otras cosas, una sobrecarga de trabajo. Por una parte, por la necesidad de conseguir empleos precarios o autogenerarse formas de ingreso informales (FRASER, 1997). Por otra parte, ante los recortes estatales en servicios y derechos, las mujeres pobres y trabajadoras se vieron en la obligación de reforzar su carga de trabajo reproductivo en los hogares y la comunidad, para garantizar cuidados básicos: alimentación, educación, salud, entre otros.

Además, esta década constituyó un momento de migraciones internas o recepción de inmigrantes de países limítrofes, entre quienes se encontraban mujeres bolivianas, peruanas, y paraguayas que se insertaron trabajando como niñeras, criadas, auxiliares de salud. Es decir, las mujeres frecuentemente racializadas se insertaron en tareas esenciales para la reproducción, invisibilizadas y en condiciones de precariedad e informalidad que no variarían significativamente hasta la actualidad (MAGLIANO, 2015).

Se cristalizó desde este momento un pasaje del Estado como garante de derechos sociales a la generación de políticas asistenciales hacia los sectores populares. Si bien, entonces, el reclamo de los sectores populares organizados, por ejemplo, en el marco del movimiento piquetero², era el trabajo "digno" o

\footnotetext{
${ }^{2}$ Compuesto por organizaciones de trabajadores desempleados, considerado como uno de los principales focos de resistencia frente al aumento del desempleo, la desigualdad, y la pobreza en el período (CROSS; PARTENIO, 2004).
} 
"genuino", las repuestas para el sector desde fines de la década de los 1990 fueron en forma de subsidios monetarios a cambio de una contraprestación laboral, como una forma de paliar la situación de vulnerabilidad de los sectores más pobres, según el modelo del empleo forzoso (workfare), dictaminado por los organismos internacionales (SVAMPA, 2009).

Durante la década de 2000, con los gobiernos neodesarrollistas (FÉLIZ; PÉREZ, 2010) encabezados por Néstor Kirchner y Cristina Fernández, operó un pasaje a nivel estatal de la política de los programas sociales mencionados a la de programas de cooperativas de trabajo que algunos autores nombran como "giro productivista" dentro de la política social (CROSS; FREYTES FREY, 2007), en combinación con la implementación de la Asignación Universal por Hijo, un programa de transferencia condicionada (PTC) que consiste en un ingreso monetario mensual de tendencia universalista para los menores de edad de familias sin trabajo registrado (RODRÍGUEZ ENRÍQUEZ, 2011).

Los programas de cooperativas, dependientes del Ministerio de Desarrollo Social, a pesar de integrar estadísticamente a las personas en el mundo del trabajo, fueron pensados e implementados como proyectos de formación para el empleo, bajo la concepción de capacitación permanente o formación continua (MDS, 2009). Desde aquí, se fundamentan los bajos ingresos, la falta de derechos laborales y que no existe una inserción posterior de las personas en el trabajo de calidad. Las políticas sociales de este período constituyen en los hechos, formas de precarización laboral, mayormente de mujeres de sectores populares, sus principales destinatarias (FÉLIZ; DÍAZ LOZANO, 2018), que combinan esta participación en las cooperativas con otras tareas laborales informales.

Las condicionalidades para percibir la AUH vinculadas a la acreditación de cumplimiento de las responsabilidades de cuidados de hijos e hijas, así como la precariedad de condiciones que impusieron las cooperativas en función de ingresos muy por debajo de la canasta básica y casi nulos mecanismos de reinserción en el mercado formal, han complicado la posibilidad de cuestionar las desigualdades sexo-genéricas (RODRíGUEZ ENRÍQUEZ, 2015).

El Estado descargó, así, nuevamente en mentes y cuerpos femeninos la responsabilidad social del cuidado. En particular, las políticas sociales 
propendieron a articular una nueva "economía de los cuidados" (RODRÍGUEZ ENRÍQUEZ, 2005) sobre las espaldas de las mujeres.

El trabajo de reproducción de la vida, feminizado y no pago, continúa funcionando en la actualidad como amortiguador de la pobreza, reemplazando y garantizando la atención de la salud, educación y alimentación de las personas en un enfoque de corresponsabilidad que en realidad implica que el Estado asigne dinero a niños con necesidades insatisfechas y les asigne la responsabilidad de su resolución a las mujeres.

Como resultado se profundiza la sobrecarga de trabajo sobre las mentes y los cuerpos de las mujeres trabajadoras que, cotidianamente, deben realizar labores remuneradas y no remuneradas dentro y fuera de los hogares para sostener sus vidas y las de sus familias. De esta forma, las desigualdades producto del capitalismo, el patriarcado, y el racismo se refuerzan en la pelea cotidiana por la supervivencia.

\section{Todos los trabajos, el trabajo}

"Hago listas en mi cabeza", "pienso en otra cosa que tengo que resolver mientras hago algo", "me organizo", "intento hacer todo", "trato de dejar todo listo antes de salir", son algunas de las frases con las que las mujeres que entrevistamos dan cuenta de la superposición y la mezcla de actividades cotidianas. A partir del seguimiento de los tránsitos diarios de las mujeres, podemos distinguir - solo a efectos analíticos - diversas formas que toma el trabajo de las mujeres para la sostenibilidad de sus vidas, sus familias, y comunidades.

Para comenzar, la totalidad de estas mujeres realizan trabajos de cuidados no remunerados en sus hogares. Como plantea Silvana Sciortino (2018), en su estudio sobre mujeres de sectores populares, a diferencia de las posibilidades de otras familias de sectores medios, las mujeres "del barrio" ( sic) cuentan con la posibilidad de acudir al mercado para encontrar formas de cuidado alternativo, por lo que establecen modalidades "creativas" familiares y colectivas que 
amortiguan los vacíos de la intervención estatal y la imposibilidad económica de acceder al mercado para satisfacer esta necesidad.

Por consiguiente, el trabajo de cuidados constituye una tarea desempeñada por las mujeres, o sus redes familiares feminizadas, o en los casos donde hay cierto reparto de tareas con integrantes varones de las familias, y la responsabilidad por su organización y cumplimiento igualmente recae sobre ellas.

Ahora bien, en el caso de las mujeres militantes, parte de este trabajo de cuidados es colectivizado, puesto en común por las mujeres en el marco de la organización, a partir, por ejemplo, de la práctica del comedor comunitario, la creación de "guarderías" o espacios de cuidados infantiles, la limpieza de espacios comunes, los roperos comunitarios, etc. Precisamente estas son las actividades comunitarias - mayoritariamente desempeñadas, o al menos, organizadas por mujeres - que permiten la reproducción de la dinámica de lo colectivo territorial y producen al Frente como un espacio cotidiano.

Además de este trabajo de cuidados familiar y comunitario, las mujeres (y también los varones), en el marco del Frente, realizan tareas remuneradas en las cooperativas de trabajo y en emprendimientos productivos. En el primer caso, se trata de una actividad con acuerdos fijos con relación a los horarios y espacios de desempeño, en la que el pago a cada cooperativista lo realiza el Estado Nacional Argentino, como parte de un programa dependiente del Ministerio de Desarrollo Social. En líneas generales, son tareas de limpieza, saneamiento y mantenimiento de espacios públicos e instituciones barriales. A pesar de estar enmarcadas en una política oficial, la relación contractual de la cooperativa se produce entre la organización y el Estado, porque se trata de puestos laborales obtenidos a través de negociaciones posteriores a movilizaciones y "planes de lucha" (en términos nativos). Esta forma de obtención de los lugares dentro de los programas permite, también, ciertos mecanismos de autogestión y negociación de los tiempos, condicionalidades, espacios de trabajo donde influyen en las necesidades femeninas en torno a los cuidados.

Los emprendimientos productivos, en cambio, son actividades grupales autogestivas, destinadas a generar algún ingreso monetario más allá de los 
programas mencionados. En los "productivos", como son denominados por sus impulsores, se trabaja "sin patrones" y generalmente se desempeñan en las ramas de alimentación, carpintería, herrería, serigrafía, costura, entre otras, e incluyen como aspecto importante la capacitación en oficios y en valores cooperativos del trabajo.

El trabajo remunerado en las cooperativas y "productivos” entra en relación con otras actividades que generalmente realizan la totalidad de los participantes - a diferencia de los cuidados colectivizados -, por fuera del espacio del comedor. Estas otras actividades, como por ejemplo la participación en las movilizaciones, en ciertas reuniones, la gestión de alimentos en el municipio, constituyen "criterios" (en términos nativos) o precondiciones para formar parte de la organización y percibir algún ingreso. En la práctica, son actividades que forman parte de, podríamos decir, "el trabajo de conseguir y mantener el trabajo remunerado" en el marco del Frente.

Pero, además, dentro de la organización, hay toda una serie de actividades de carácter completamente voluntario - no son condiciones para la pertenencia o para percibir remuneración dentro de la organización -, en las que participan sólo algunas personas. Estas son, por ejemplo, los talleres de formación en salud, en política, en géneros; las reuniones con los otros núcleos barriales de la organización; las acciones culturales de encuentro y recreación colectiva; las discusiones políticas e iniciativas de apoyo a ciertas luchas; las reuniones de relaciones políticas con otras organizaciones; y todo un conjunto de tareas de coordinación con instituciones barriales y vecinas. Estas actividades, desde las integrantes del Frente, son caracterizadas en todos los espacios con el nombre de "militancia" o "tareas militantes" y la participación en ellas es optativa.

Por último, la gran mayoría de las participantes entrevistadas realiza trabajo remunerado por fuera del encuadre colectivo para complementar el ingreso obtenido en el marco de la organización, o viceversa, del cual el ingreso cooperativo resulta complemento. En la mayoría de los casos, es un trabajo basado en tareas domésticas, y se produce en situaciones de precariedad y falta de derechos laborales formales. En general, se trata de trabajo de cuidados pago 
que realizan en otras casas, limpiando, atendiendo personas ancianas, enfermas o cuidando niños y niñas.

Esta multiplicidad de tareas recayendo sobre el tiempo y el cuerpo femeninos suponen un trabajo físico y mental que no cesa y que en gran medida se desarrolla uniendo espacios: entre la casa, el comedor, el barrio, la escuela, el empleo en otras casas, la calle. El trabajo aparece como un proceso continuo en el tiempo y en el espacio, un conjunto de actividades entrelazadas y hasta superpuestas que incluye labores asalariadas o remuneradas, actividades de cuidados domésticos y comunitarios y tareas ligadas a la participación política. Un continuo de trabajo organizado fundamentalmente en función de otros y cuyos tiempos fluctúan de acuerdo con el contexto económico, social y político y también a las iniciativas individuales y colectivas desplegadas por las mujeres.

\section{El trabajo de la participación comunitaria}

Las mujeres participantes en esta investigación, como parte de la búsqueda de sostener sus vidas y las de sus familias, han decidido participar en una organización social y política asentada territorialmente. Aquí, consideramos que la integración en organizaciones sociales, políticas, religiosas, barriales, entre otras, es parte de un conjunto de tácticas (en términos de DE CERTEAU, 1986) de sostenibilidad de la vida que las mujeres configuran en el quehacer diario; ellas realizan estas participaciones colectivas en algunos casos, reconciliando en los tránsitos cotidianos prácticas e idearios institucionales diferentes y hasta contradictorios, estableciendo relaciones de intercambio, conflicto y cooperación que configuran lo común (GUTIÉRREZ; NAVARRO; LINSALATA, 2016; LINSALATA, 2016).

Con la integración al trabajo comunitario en el marco de una organización como el Frente que plantea "criterios" de participación, algunas mujeres señalan que al tiempo que les permite resolver el sustento básico, comienzan a tener una nueva exigencia. 
Y entrando a la organización, me parece que un cargo más me hiciera, aparte de ser mamá, ama de casa, aparte me agarré otro cargo, o una responsabilidad más. No sólo me ocupo de ama de casa, sino que como estoy asistiendo a una organización, obligaciones que hay que hacer, por ahí apoyar, como en otros momentos vi que apoyaron otros compañeros, aunque sea con la presencia, o acompañar o ir aprendiendo cómo. Acercarse a una gestión (reunión con funcionarios), todo eso quiero aprender. No siempre por la obligación nomás voy a las reuniones y las marchas, sino que quiero aprender y también acompañar o agradecer a los otros compañeros que hayan luchado más antes, que por ellos tuvimos cooperativas o planes. [...] O que todo es posible luchando u organizándose, todo es posible. (Entrevista a Margarita, Villa Argüello, 30 mar. 2015)

Tal como se lee, para Margarita, compatibilizar las actividades diarias implicadas en la maternidad, la cooperativa, el comedor se vuelve un desafío cotidiano signado a la vez por sentimientos de obligación y deseo.

En una charla informal con otra participante, Luz, sin que mediara una pregunta específica, ella había comentado:

Le dedico más de 8 horas diarias a la organización. (Nota de campo, Comedor Juanito Laguna, Villa Argüello, 25 oct. 2014)

Esta referencia une la alusión a un período de tiempo ligado al trabajo remunerado y registrado (8 horas) con las tareas que realiza en la organización. Sin embargo, detrás de esta frase hay una idea amplia de trabajo: se refiere a las diferentes tareas que desempeña en el barrio, más allá de las horas dedicadas a trabajo retribuido monetariamente a partir del movimiento en las cooperativas de trabajo. Cuando reconstruimos junto a Luz la distribución de tareas y tiempos cada día, vemos que ésta dista mucho de estar organizada en jornadas delimitadas. Aquella referencia a las "8 horas" de trabajo, en el marco de la organización, alude a un imaginario de jornada de empleo formal, pero que en la práctica constituye una sumatoria aproximada de tiempos destinados a tareas entrecortadas y superpuestas con otras. Para citar algunos ejemplos: mientras que retira a su hijo del colegio, realiza trabajo comunitario, porque contacta a otras vecinas para invitarlas a cocinar al comedor, cuando realiza apoyo escolar para los niños y las niñas del barrio también ayuda con la tarea a su hijo. Aquí, el cuidado de su hijo se realiza en parte dentro de su casa, pero también en las instituciones barriales y en los ámbitos colectivos del Frente. De igual manera, al 
Triple presencia femenina en torno a los trabajos: mujeres de sectores populares, participación política y sostenibilidad de la vida

Juliana Díaz Lozano

realizar trabajo remunerado en la cooperativa, también está participando del mantenimiento de las calles en que vive.

Esta superposición o solapamiento de actividades, tiempos, y espacios se visualiza con claridad cuando las mujeres relatan sus tránsitos, como lo hace a continuación Margarita:

Arreglarme el día se me hace (sic) complicado a veces. Por ahí los dias de las marchas se me hace complicado, por ahi un poco dificil de organizarme. En la mañana, por ejemplo, tengo que llevar este año, un cargo más tengo, llevar a mi hijito o retirarlo del jardín. A la vez, a las 8 de la mañana va al jardín, y tengo que estar al pendiente a ver si no me llaman del jardín. Eso tengo eso en mi cabeza, aparte de ir a trabajar y volver 11 o 12 del mediodía, como tengo acá el quiosquito también tengo otra responsabilidad también. Luego a ir a hacer compras. Ir y volver y como sea a las 12:30. Voy a comprar sola en la moto. Yo todavía no sé manejar la camioneta, entonces me lo trato de arreglar con la moto solamente, por ahi voy día por medio o todos los dias si me falta algo. Y tengo que volver de dónde sea para las 12:30 porque mis dos hijos mayores a esa hora salen para la escuela. Y como yo llego a los dos chiquititos, tengo que llegar si o sí, sea de una reunión del Frente, de una gestión o de una compra, tengo que regresar, acá. Y después ya tengo que ver en la tarde de hacer mis cosas de la casa y los viernes la movilización, un poco se me complica porque tengo que ver de no hacerle faltar mucho a la escuela a mi hijo y a la vez quiero ir a la marcha, ahi se me divide un poco, porque tengo dos obligaciones. Trato de resolverlo, por ahi me los llevo a los chiquitos y si puedo dejarlos con alguien, voy. Trato de resolvérmela así. Estar en una organización también es una responsabilidad más, pero aprendes de ahí, también es un beneficio para que aprendas más tú, abrirte más, un poco mejorar tu vida. (Entrevista a Margarita, comedor Madres Unidas, 30 mar. 2015)

El testimonio de Margarita da cuenta de una modalidad de organización del trabajo donde no se distingue con claridad el trabajo productivo del reproductivo, ni los espacios internos o íntimos y los públicos, y dónde la respuesta a las condiciones de precariedad vital (PÉREZ OROZCO, 2014) ${ }^{3}$ es la táctica de la mezcla y el solapamiento de tareas.

3 Según la autora, la idea de precariedad vital contiene (y a la vez desborda) la de precariedad laboral. Esta última importa por la combinación de sus efectos con todos los demás factores que median en el nexo entre calidad de vida y salario (servicios públicos, redes sociales, bienes comunes, trabajos no remunerados) en el marco de unas determinadas aspiraciones vitales. Incluye un elemento de incertidumbre generalizado que repercute en los arreglos vinculados a los cuidados. 
Es decir, en estas experiencias femeninas, la sobrecarga de trabajo es una situación permanente, a veces asumida como inevitable, "siempre es así, las mujeres tenemos que hacer todo", y con frecuencia aludida con los términos "esfuerzo", "sacrificio", "calvario", entre otros. A partir de las entrevistas, donde les pedimos que realizaran un relato pormenorizado de las actividades rutinarias de cada día, pudimos notar en las mujeres reacciones de azoramiento en relación con el volumen de trabajo que realizan y expresar "a veces te dan ganas de llorar y de tirar todo 'a la miércoles", o "se me hace muy complicado organizar todo a veces". La expresión utilizada con frecuencia es "tengo que", aludiendo a deberes que se explican fundamentalmente en las obligaciones de cuidados por tener personas a cargo tanto en los ámbitos familiares como barriales. Esto ocurre más allá del lugar de la presencia de los varones adultos en las familias (p. ej., Margarita vive con su marido), porque la organización del trabajo diario se produce desde modelos monoparentales del cuidado (SCIORTINO, 2018), a partir de los cuales las mujeres son las responsables últimas de la organización del sostenimiento doméstico.

Esto se relaciona, por una parte, con que las mujeres acceden a los trabajos más flexibles y precarios (situación no alterada por las políticas sociales de las cuales son destinatarias privilegiadas), pero también porque, a pesar de trabajar parte del tiempo fuera de la casa, el trabajo doméstico y de cuidados sigue recayendo sobre sus cuerpos (FÉLIZ; DÍAZ LOZANO, 2018). Esta dinámica intra-hogares, se traslada - en parte - a las tareas de reproducción de la vida comunitaria y en las organizaciones, donde las mujeres garantizan mayoritariamente la alimentación, la vestimenta, los cuidados infantiles, el arreglo de infraestructura y mantenimiento barrial, la asistencia a reuniones organizativas, entre otras actividades diarias.

\section{Más allá de las jornadas laborales: las presencias femeninas}

La idea de "jornadas laborales" de las mujeres, trabajada desde las perspectivas feministas de la economía, tiene un profundo valor explicativo y político, que, al tiempo que vuelve visible los trabajos ocultos y no pagos realizados por las mujeres, da cuenta de la desigual división del trabajo sexo- 
genérico en la sociedad y la sobre carga específica sobre mujeres del pueblo trabajador. Para Rodríguez Enríquez (2015), por ejemplo, la multiplicación de las jornadas para las mujeres es el "vector de reproducción de las desigualdades sociales". Entonces, la visibilización de las "jornadas" tiene la importancia innegable de señalar la desigual división por género del trabajo en la sociedad y de abrir la mirada al abordaje de los usos del tiempo como herramienta para intentar cuantificarla.

Sin embargo, como advertimos a partir del análisis de las experiencias vitales de las mujeres de sectores populares en relación con el trabajo, no solemos encontrar momentos estancos y espacios definidos, es decir, por ejemplo, tiempo destinado al trabajo remunerado separado con claridad de aquel en que se realiza trabajo de cuidados o comunitario. De similar forma ocurre con los espacios, donde la dicotomía trabajo remunerado fuera del hogar $\times$ trabajo no remunerado dentro del hogar es reemplazada por tránsitos flexibles que las mujeres organizan día por día. Entonces, a pesar de la importancia política que tuvo la incorporación de la idea de doble jornada para pensar el trabajo femenino en el ámbito doméstico y en el mercado, esta denominación no describe cabalmente la forma en que las mujeres trabajadoras y de sectores populares vivencian el trabajo en la actualidad.

Para poder visibilizar la multiplicidad y superposición de actividades, espacios y temporalidades del trabajo femenino en el capitalismo tardío, teóricas italianas, como Laura Balbo, Chiara Saraceno y Antonela Picchio, acuñaron el término de doble presencia (SCIORTINO, 2018). De esta manera, la distinguen de la doble jornada, que refiere a la suma de dos o más empleos de manera secuencial y no superpuesta, vinculada al concepto de pluriempleo. La doble presencia de las mujeres supone, para ellas, "la nueva forma de división sexual del trabajo en el capitalismo avanzado" (CARRASQUER OTO, 2009, p. 61). Esta se manifiesta en una vida cotidiana donde varones y mujeres están presentes en el mercado laboral y donde el trabajo doméstico continúa a cargo de las mujeres, en buena medida, pero no exclusivamente, como trabajo privado. Esta doble presencia supone la reconstrucción de una identidad femenina a medio camino 
Triple presencia femenina en torno a los trabajos: mujeres de sectores populares, participación política y sostenibilidad de la vida

Juliana Díaz Lozano

entre estas pertenencias que deben compatibilizarse en la cotidianeidad (CARRASQUER OTO, 2009).

Por consiguiente, este concepto resulta más pertinente para nombrar la vinculación de las mujeres con el trabajo en la actualidad, caracterizada por la acumulación y mezcla en espacio y tiempo de trabajos con lógicas distintas. La doble presencia femenina se asienta en la tarea cotidiana de combinar de manera flexible el trabajo en el mercado, asalariado, con las actividades reproductivas domésticas, muchas veces ni siquiera visibilizadas socialmente como trabajo. Justamente, son las características del trabajo reproductivo las que distinguen la doble presencia de la doble jornada: la sincronía, es decir, la reiteración circular de actividades cotidianas durante el ciclo de vida, la disponibilidad hacia el resto de las personas, y la simultaneidad de actividades. Estas cualidades de la doble presencia generan, a su vez, la dificultad de cuantificar el tiempo de trabajo de las mujeres, que se vuelve un continuo organizado en pos de las necesidades de otras personas.

Como vimos en los testimonios de Luz y Margarita, en las experiencias de las mujeres, cada día se experimenta como una gran jornada laboral, con tareas entrelazadas y preocupaciones superpuestas en tiempo y lugar. Para ellas, la forma de lidiar con el gran volumen de tareas es: la mezcla y la sobrecarga; el correr de un lado hacia el otro; el cuidar sin salario al tiempo que cuidan por un ingreso; el cocinar para las familias del barrio, al tiempo que cobran un "plan social"; irse de la asamblea a las apuradas para bañar a uno de sus hijos y volver para el final con el niño cargado; ir a la movilización con uno de sus hijos y repasar los deberes del colegio mientras se espera la respuesta de la gestión... Coincide con lo planteado por Federici (2013, p. 42) como "el aprieto [...] de que dependan de nosotras las vidas de otras personas y la imposibilidad de ver dónde comienza y termina nuestro trabajo, dónde comienzan y acaban nuestros deseos".

Para quienes trabajan de noche cuidando personas a cambio de una remuneración, por ejemplo, y participan comunitariamente, se vuelve complejo incluso delimitar el inicio y final de los días, debido a que el momento del descanso es la variable de ajuste que puede moverse de acuerdo con el resto de las actividades barriales o del Frente, no siempre regulares en la semana. En 
todos los casos, el trabajo constante es el de organización y gestión del tiempo de trabajo, es decir, las tácticas de combinación de actividades para resolver la vida cotidiana, en la encrucijada entre producción y reproducción. Esta gestión del tiempo es, siempre, la actividad más difícil de delegar para las mujeres.

Pensar esta situación desde la idea de doble presencia permite relativizar las dicotomías que estructuran el trabajo en el capitalismo fordista: presencia/ausencia; público/privado; trabajo/no trabajo; productivo/reproductivo (CARRASQUER OTO, 2009). Y entender cómo la mayor flexibilidad en los tiempos y espacios de trabajo en nuestros contextos se traduce en un mayor esfuerzo por parte de las mujeres para poder organizar y gestionar la vida cotidiana. Esto se profundiza y potencia en las experiencias de las mujeres de sectores populares, debido a las situaciones de precariedad vital.

\section{Potencialidades de pensar la triple presencia de las mujeres militantes}

Ahora bien, ¿qué sucede con el trabajo femenino con la integración a un marco colectivo y comunitario, en este caso al Frente? Cuando miramos integralmente el trabajo de las mujeres, el asalariado, doméstico, cooperativo y militante, vemos que esta multifuncionalidad femenina afecta la disponibilidad de tiempos propios. En este marco, la participación política comunitaria es vivenciada como una nueva actividad a compatibilizar, "una nueva obligación" además del trabajo doméstico y el remunerado -, con lo que podemos hablar de una triple presencia en el caso de las mujeres militantes.

Pero, al mismo tiempo que implica más responsabilidades, y paradójicamente, esta triple presencia es una táctica que las mujeres configuran para lidiar con la sobrecarga y con el lugar que la división por género del trabajo asigna a los múltiples trabajos femeninos. Entonces, la integración a un colectivo comporta, para las mujeres de este estudio, una opción positiva de intento de resolución de las problemáticas cotidianas. La decisión de permanecer en una organización que plantea "un cargo más", "hacer de todo", "resolvérselas" a cambio de ingresos magros se explica, en parte, por las relaciones sociales que se establecen cotidianamente, por el espacio de sociabilidad y confianza que se 
conforma para la resolución de necesidades. Pero, además, como dijimos, los espacios allí generados son lugares de gestión colectiva de cuidados, que permiten articular, mezclar y yuxtaponer actividades "reproductivas" y “productivas", pagas y no pagas, en tácticas dinámicas, desde las cuales las mujeres negocian el uso del tiempo que realizan con las otras mujeres y los varones de la organización, pero también con integrantes de sus familias.

Además, esta tercera presencia femenina en los ámbitos comunitarios tiene la importancia de visibilizar y jerarquizar los cuidados que de otra forma se resolverían en espacios íntimos y familiares. Estos cuidados colectivizados instituyen nuevos ámbitos abiertos al barrio, como jardines, guarderías, talleres, bachilleratos, escuelas autogestionadas, roperos comunitarios, entre tantos otros, que visibilizan las necesidades de cuidados. A su vez, la colectivización entre mujeres les permite reconocer al trabajo de cuidados en términos de interdependencia (PÉREZ OROZCO, 2014), como un trabajo que sostiene la vida cotidiana de los sectores populares desde una lógica donde lo monetario y la ganancia no son centrales. Para las mujeres, esta triple presencia está cargada con la potencia de constituir un saber y una herramienta para la construcción cotidiana de formas de organización comunitaria.

A partir de esta triple presencia, las mujeres entran y salen de los diferentes ámbitos horadando las fronteras entre lo público y lo privado, lo colectivo y lo individual, lo personal y lo político. La participación en el Frente, como parte de estas tácticas multiformes comunitarias, permite a las mujeres colectivizar una parte del trabajo de cuidados, dándole una nueva visibilidad y generando una posibilidad de encuentro y organización femenina desde lógicas de cooperación antagónicas con la individualización del capital y el aislamiento que promueve, al decir de Amaia Pérez Orozco (2014), una ética reaccionaria de los cuidados. Estas prácticas compartidas de cuidados pueden constituir una base para el cuestionamiento de la división por género del trabajo, debido a que la colectivización desvela la dimensión política de los cuidados, es decir, su organización ya no es pensada desde una asignación naturalizada, sino a partir de la deliberación cotidiana. Justamente es en aquella cotidianeidad en tanto terreno político, público y lugar de transformación y (re)producción de las 
Triple presencia femenina en torno a los trabajos: mujeres de sectores populares, participación política y sostenibilidad de la vida

Juliana Díaz Lozano

relaciones existentes (VARGAS, 2008) donde puede rastrearse situadamente estos deslizamientos.

La participación política que realizan estas mujeres constituye en sí una práctica disruptiva para las lógicas patriarcales. El modo en que - cada una singularmente, pero en colectivo - logra lidiar con la triple presencia les permite conquistar espacios y tareas fuera del canon patriarcal que requiere que las mujeres estén sujetas en una única narrativa íntima. Su participación es en sí misma un hecho subversivo, porque no se trata de una declamación o un posicionamiento escrito, en la densidad de las prácticas que traman cotidianamente uniendo espacios, yendo y viniendo incansablemente, tramando relaciones corporeizadas que las hacen más fuertes, y también enfrentando incomodidades, tensiones, construyendo común donde la propuesta hegemónica es la casa y el aislamiento.

La potencia de pensar en términos de múltiples presencias femeninas reside, entonces, en que nos permite visualizar en la cotidianeidad los condicionamientos que las relaciones de género y clase imponen, pero también abordar y valorizar las tácticas femeninas que inauguran, desde la sobrecarga y el solapamiento, nuevas posibilidades, territorios y modos de organización para la sostenibilidad de la vida individual y colectiva.

\section{Referências}

ANDÚJAR, Andrea. Rutas argentinas hasta el fin. Mujeres, política y piquetes 1996-2001. Buenos Aires: Luxemburg, 2014.

CARRASCO, Cecilia. Cuaderno Bolteneano. Buenos Aires: Cátedra Virginia Bolten/Centro Interdisciplinario para el Estudio de Políticas Públicas y Sociedad de Economía Crítica, 2017.

CARRASQUER OTO, Pilar. La doble presencia. El trabajo y el empleo femenino en las sociedades contemporáneas. Directora: Teresa Torns Martín. 2009. 217 h. Tesis (Doctorado en Sociología) - Universidad Autónoma de Barcelona, Barcelona, 2009. 
Triple presencia femenina en torno a los trabajos: mujeres de sectores populares, participación política y sostenibilidad de la vida

CAUSA, Adriana. La configuración de la trama de mujeres desocupadas (piqueteras). La acción colectiva antes, durante y después de los piquetes. In: CONGRESO EUROPEO DE LATINOAMERICANISTAS, 5., Bruselas. Anales [...]. Bruselas: [s. n.], 2007.

CROSS, Cecilia; PARTENIO, Florencia. Mujeres y participación: las organizaciones piqueteras y las relaciones de género. In: CONGRESO NACIONAL DE SOCIOLOGíA, 2., 2004, Buenos Aires. Anales [...]. Buenos Aires: [s. n.], 2004.

DE CERTEAU, Michel. Usos y tácticas de la cultura ordinaria. Revista Signo y Pensamiento, Bogotá, v. 5, n. 9, p. 23-30, 1986.

FEDERICI, Silvia. Revolución en punto cero. Trabajo doméstico, reproducción y luchas feministas. Madrid: Traficantes de Sueños, 2013.

FÉLIZ, Mariano; DÍAZ LOZANO, Juliana. Trabajo, territorio y cuerpos en clave neodesarrollista. Argentina, 2002-2016. Perfiles Latinoamericanos, México, v. 26, n. 52, p. 1-26, 2018.

FÉLIZ, Mariano; PÉREZ, Pablo. Políticas públicas y las relaciones entre capital y trabajo.

Contrastes y continuidades en la posconvertibilidad a la luz de la historia argentina. In:

FIGARI, C.; LENGUITA, P.; MONTES CATÓ, J. (ed.). El movimiento obrero en disputa. La organización colectiva de los trabajadores, su lucha y resistencia en la Argentina del siglo XX. Buenos Aires: CICCUS/CEIL PIETTE, 2010. p. X-y.

GUTIÉRREZ, Raquel; NAVARRO, Mina; LINSALATA, Lucía. Repensar lo político, pensar lo común. Claves para la discusión. Puebla: Benemérita Universidad Autónoma de Puebla, 2016. (Documento de Cátedra Seminario Entramados Comunitarios y Formas de lo Político).

LINSALATA, Lucía. Cuando manda la asamblea: lo comunitario-popular en Bolivia - una mirada desde los sistemas comunitarios de agua de Cochabamba. México, DF: Sociedad Comunitaria de Estudios Estratégicos, 2015.

PÉREZ OROZCO, Amaia. Subversión feminista de la economía. Madrid: Traficantes de Sueños, 2014.

\footnotetext{
REGUILLO, Rosana. La clandestina Centralidad de la Vida Cotidiana. En Lindón Villoria, (coord.) La vida cotidiana y su espacio-temporalidad (pp. 77-94). Madrid: Anthropos, 2000.
} 
RODRÍGUEZ ENRÍQUEZ, Corina. Programas de transferencias condicionadas de ingreso e igualdad de género. ¿Por dónde anda América Latina? Santiago de Chile: CEPAL, 2011.

RODRÍGUEZ ENRÍQUEZ, Corina. El trabajo de cuidado no remunerado en Argentina: un análisis desde la evidencia del Módulo de Trabajo no Remunerado. Buenos Aires: Equipo Latinoamericano de Justicia y Género, 2015. (Serie Documentos de Trabajo: Políticas Públicas y Derecho al Cuidado, No. 2).

SCIORTINO, Silvana. El trabajo de cuidado entre las titulares del programa social "Ellas Hacen": una etnografía sobre arreglos familiares, leonas y mujeres superpoderosas. Cuadernos de Antropología Social, Buenos Aires, n. 48, p. 5571, 2018.

VARGAS, Virginia. Nuevas formas de participación política y de luchas feministas en lo local y en lo global en el nuevo milenio. In: CONGRESO IBEROAMERICANO DE ESTUDIOS DE GÉNERO, 4., 2008, Rosario. Anales [...]. Rosario: [s. n.], 2008. 